\title{
IMAGE DENOISING USING WAVELET TRANSFORM
}

\author{
Sameer Khedkar ${ }^{1}$, Kalyani Akant ${ }^{2}$, Milind M. Khanapurkar ${ }^{3}$ \\ ${ }^{1}$ Research Scholar, Electronics and telecommunication Department, GHRCE, Nagpur, Maharashtra, India \\ ${ }^{2}$ Associate Professor, Electronics and Telecommunication Department, GHRCE Nagpur, Maharashtra, India \\ ${ }^{3}$ Professor, Electronics and Telecommunication Department, GHRCE, Nagpur, Maharashtra, India
}

\begin{abstract}
Image denoising is a noise removal technique used to remove noise from noisy image. The wavelet is one of the most popular techniques in recent developments in image denoising. It is effective in denoising because of its energy transformation ability to get wavelet coefficients. It is not possible to get noise suppression and characteristics preservation of the image at the same time. In this paper an improved method is presented by which the optimal threshold for every sub-band in neighboring window is determined by Stein's Unbiased Risk Estimator (SURE). Then, the neigh shrink is applied in the neighboring window to get optimal PSNR (Peak Signal to Noise Ratio). The main aim of this research work is to increase the PSNR of an image while keeping the Mean Square Error (MSE) low. The algorithm was tested on various images and the results for different PSNR and MSE values are presented in this research paper.
\end{abstract}

Keywords: Wavelet, Denoising, Threshold, Neigh, SURE.

\section{INTRODUCTION}

The image is said to be more expressive than thousand words. The noise which corrupts the image is one of the fundamental problems to have emerged in the field of image processing, so an entire image denoising field was created within the image processing domain so as to combat the noise in the image. The noise can get introduced in the image in stages of image acquisition, compression and transmission. There are many other causes too, like hardware faults in the camera lens, lesser processing power etc. This noise introduced in image produces undesired elements in image which are not soothing to the human eye so it is a priority to reduce the noise in image to as low as possible. The domain which deals with the noise elimination is known as image denoising.

In the last decade or so the image denoising has acquired tremendous attention due to its usability in the nowadays camera machines in real time domain to produce more and more fine and clear pictures. If such processing is linked with a fine lens camera then the pictures taken will be of fine quality with lesser amount of noise. In denoising the effort is, to recover some of the lost elements in the picture due to noise but at the same time preserving or enhancing the actual picture signal carrying elements.

There are different methods invented for image denoising. In the recent years the wavelet transform emerged as an option to the formerly used Fourier Transform (FT) and its co-transforms, namely, the Discrete Sine Transform (DST) and the Discrete Cosine Transform (DCT). Wavelet transform provided some excellent localization property as well as some compact energy packet reduction. So, it became one of the most used methods with its other variants like curvelet transform and contourlet transform.

\subsection{Related Work}

During the last decade or there has been immense research in the field of image denoising using different techniques for wavelet packet. The pioneers would be the Mallat S.[1] who demonstrated that wavelet transform is particularly suitable for the applications of non stationary signals which may spontaneously vary in time. Other wavelet denoising methods depend on wavelet transform scale correlation between the wavelet coefficients[2]; Donoho and John Stone pioneered the thresholding by coining soft-threshold and hard-threshold wavelet denoising methods[3],[4],[5]..

Nowadays research in wavelet transform has gone in specific areas like in aerial images captures by airplane, SONAR images captured by submarines. Medical images like MRI can also be denoised using the wavelet transform implementing different techniques for the different picture types. During the different image processing steps, images are processed in different techniques like compression and acquisition. During these steps unwanted image elements may get introduced in the image. The nature of the noise is one of the most important prerequisite for image denoising which facilitates the further process of denoising.

\subsection{Noise in Image}

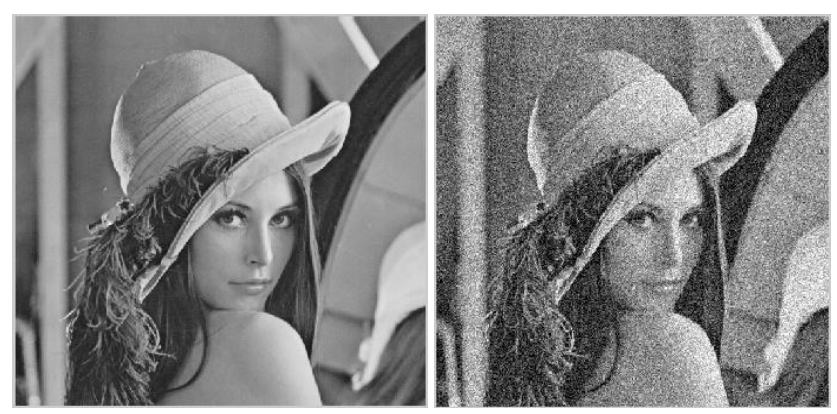

Fig -1: Clean and Noisy Image 
The noises in picture can be described as unwanted elements entered in the image during different phases of image processing. This noise can be divided into two types which are additive noise and multiplicative noise. This nomenclature is based on the mathematical nature and behavior of these noises. The additive noise will have addition to the signal whereas the multiplicative noise will be present in form of multiplication with the image signal. In the image denoising process, the nature of the noise which gets introduced in system plays pivotal role in denoising.

The additive noise is given by,

$\mathrm{t}(\mathrm{a}, \mathrm{b})=\mathrm{r}(\mathrm{a}, \mathrm{b})+\mathrm{m}(\mathrm{a}, \mathrm{b})$

The multiplicative noise is given by,

$\mathrm{t}(\mathrm{a}, \mathrm{b})=\mathrm{r}(\mathrm{a}, \mathrm{b}) \times \mathrm{m}(\mathrm{a}, \mathrm{b})$,

Where, $r(a, b)$ is the basic information signal, $\mathrm{m}(\mathrm{a}, \mathrm{b})$ is the noise entered into the original signal which gives out the corrupted noisy image $t(a, b)$. (a, b) represents the coordinates of the pixel.

\section{a) Gaussian Noise}

Gaussian nature noise is evenly spaced over the image signal. It is one of the most common noises found. Since the gaussian noise is additive noise, each pixel which is corrupted with noise is the addition of image signal with the gaussian noise. As the name suggests that, this kind of noise has Gaussian distributive nature, which has inverted circular curve shaped function.
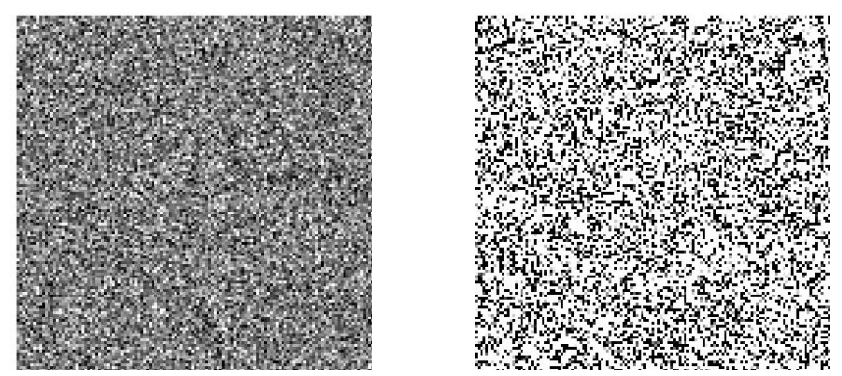

Fig -2 : Gaussian Noise with different values of mean and variance

\section{b) Salt and Pepper Noise}

Salt and pepper noise is an impulse type of noise or spontaneous noise, which is also represented as intensity spikes. This noise gets introduced in picture mainly due to anomalies in data transmission. It has only two possible values like a binary. The noisy pixels are set alternatively to the minimum or to the maximum value thus giving the image a "salt and pepper" like appearance like spreads. Unaffected pixels remain constant. The salt and pepper noise is the combination of salt noise and pepper noise ranging of values from 0 to 255 . Some of the causes of salt and pepper noise can be attributed to the fault of pixel elements in the camera hardware like sensors or lens error which may give rise to salt and pepper noise.

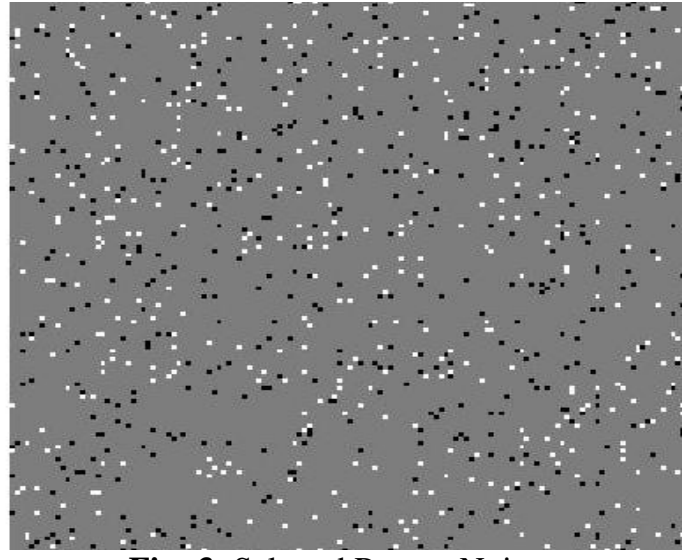

Fig -3: Salt and Pepper Noise

\section{c) Speckle Noise}

This is one of the common noise found in scientific images like the ground detection images taken from sky. The echoes which are produced in between the common random interferences is said to be the cause of this noise. Speckle noise is represented as dotted pattern as shown in Fig. 4.

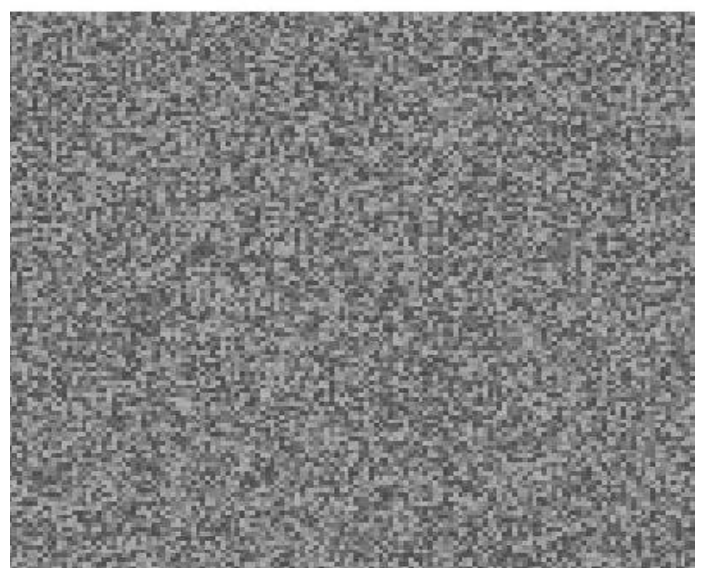

Fig- 4: Speckle Noise

\section{WAVELET TRANSFORM}

The wavelet transform's property of energy transformation comes handy in denoising. The wavelet transform has Energy compactness property which could contain most of the signal energy in a few large wavelet coefficients thus quantizing energy in which a small portion of the energy is spread across a big number of little wavelet coefficients. These types of coefficients will show details but at the same time it is also marred with high frequency noise. By congruously thresholding these selective wavelet coefficients, the image denoising is done while preserving fine details in the image. The other properties of the wavelet transform that help in the image denoising are sparseness, clustering, and correlation between neighboring wavelet coefficients. The wavelet coefficients of natural images are sparse.

Wavelet transform decomposes the image into several components based on the frequency characteristics of the signal. Wavelet transform converts the signal information 
bits in the frequency showing coefficients which are distributed in horizontal, vertical and diagonal parts of the image. These are known as the decomposed frequency components of the under observation image. This wavelet is an efficient means of spreading the energy of image. The multi resolution analysis can be done by wavelets which is one of the advantages of the wavelet transform.

Wavelets show best results for the localized details like edges and curves. In wavelet transform, the basic image is transformed into four elements also known as sub-bands namely LL, HL, LH, and HH as shown in the Fig. 7. The LL piece or sub-band is known as the approximation or average of the original image. The other three sub-bands are known as details representing components of wavelet coefficients namely the vertical details, diagonal details and horizontal details respectively.

The wavelet transform can be divided into the three components. The first is image decomposition in which an image is divided into its relative sub bands. Then it undergoes wavelet thresholding which will select and parse selected wavelet coefficients or modified ones and the last one is the inverse wavelet transform to reconstruct the original image.

\subsection{Wavelet Decomposition}

The wavelet decomposition is the first step for the image denoising using wavelet transform algorithm. The DWT (Discrete Wavelet Transform) follows the rule of hierarchy system where the sub-components are represented in the form of frequency tiers. Image is reduced and converted into four constituent by applying corresponding wavelet transform . These sub bands are produced by application of horizontal and vertical filters as shown in Fig.5. The label like HL1, HH1 and LH1 correspond to details representing coefficients while sub-band LL1 represents rough level coefficients [6] [7]. The LL1 sub band is again decomposed to get 2D decomposition as shown in Fig. 7.

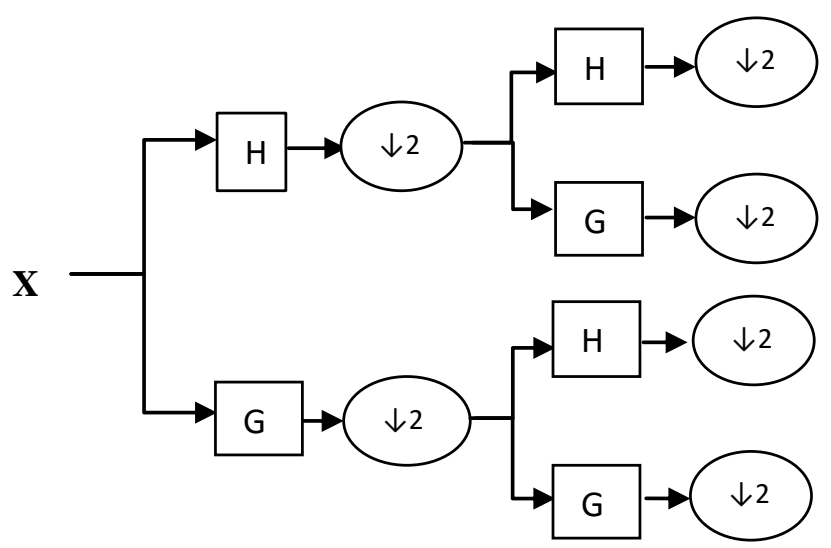

Fig- 5: 2D decomposition where $\mathrm{G}$ denotes high pass filter and $\mathrm{H}$ denotes low pass filter

\begin{tabular}{|c|c|}
\hline Image & Horizontal \\
Approximation & Detail \\
LL & $\mathrm{HL}$ \\
\hline Vertical & Diagonal \\
Detail & Detail \\
LH & HH \\
\hline
\end{tabular}

Fig- 6: A basic element figure for 1D decomposition

\begin{tabular}{|c|c|c|c|c|}
\hline \multirow[t]{2}{*}{ LL1 } & \multirow[t]{2}{*}{ HL1 } & LL2 & HL2 & \multirow[t]{2}{*}{ HL1 } \\
\hline & & LH2 & HH2 & \\
\hline LH1 & HH1 & LH1 & & HH1 \\
\hline
\end{tabular}

Fig- 7: 1D and 2D decomposition

The $\mathrm{L}$ and $\mathrm{H}$ in the Fig. 6 and 7 denote the low frequency signal and high frequency signal respectively. To get 2D decomposition, the wavelet transform is again applied to LL1. The 1D decomposed image has four sub-bands as shown in Fig 6. First is the approximation or averaging, second is the horizontal detailed coefficients having the frequency component high and low, third is the diagonal detail having both frequency components as high and then fourth one is the vertical detail having frequency components as low and high clockwise as shown in Fig 6. For $2 \mathrm{D}$ decomposition the DWT is again applied only to approximate details to get further decomposition level and so on as shown in Fig. 7.

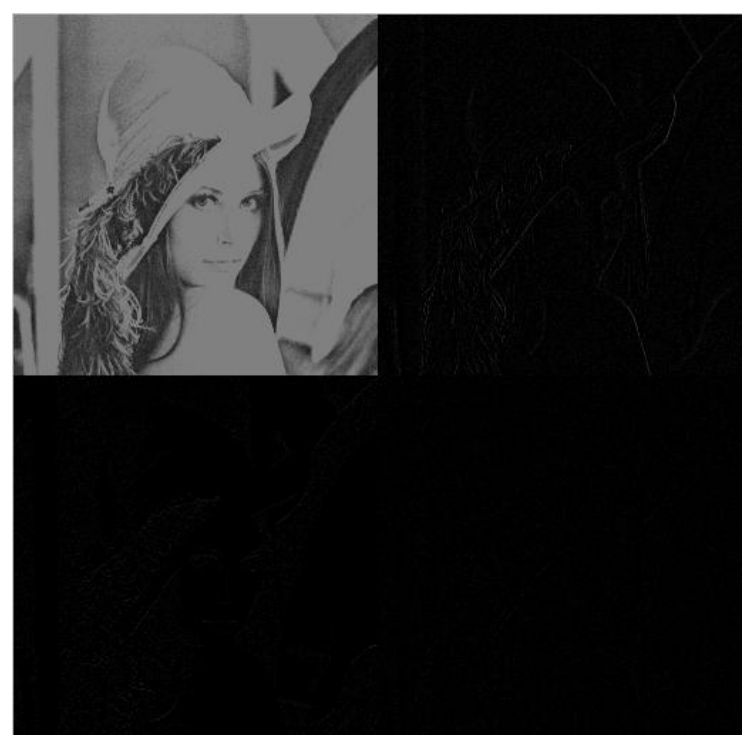

Fig- 8 : Decomposed Lena Image (1D) 


\section{DENOISING ALGORITHM PROPOSED}

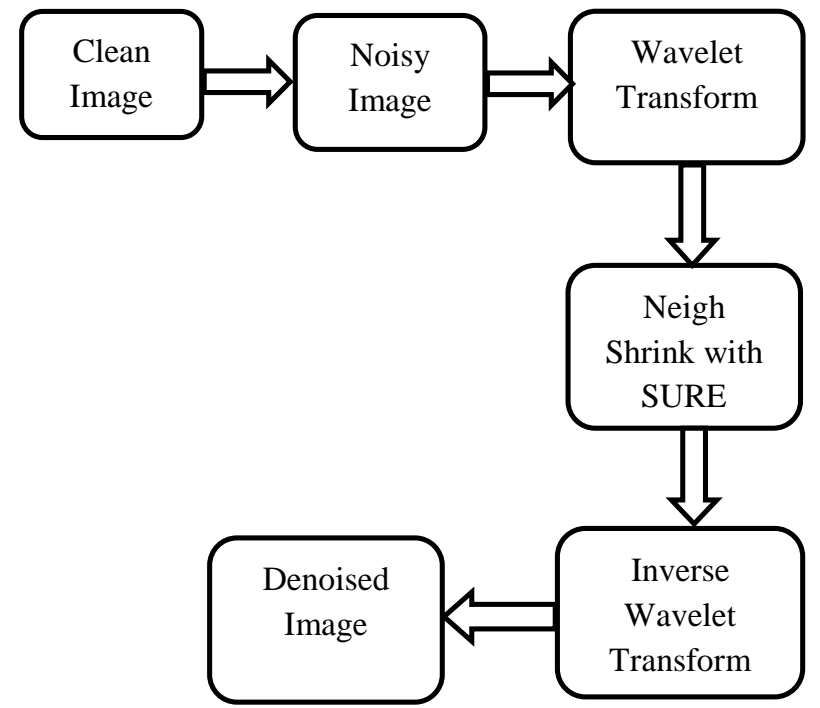

Fig- 9: Proposed Algorithm for wavelet denoising with neigh shrink

The algorithm in Fig. 9 shows the basic steps that will be followed in the implementation of image denoising algorithm. First the clean image will be corrupted with the Gaussian value according the parameters like the variance and mean. The clean image will then carry Gaussian noise function with it and the picture elements will be corrupted with noise producing undesired effects in the picture.

Then the discrete wavelet transform is applied to the noisy image which gives the wavelet decomposition of the noisy image. The decomposed image will have primarily four sub band images. The four sub bands are average or approximate of image, vertical, horizontal and diagonal detail. For 2D decomposition the approximate image again undergoes the wavelet transform to get four sub-bands again.

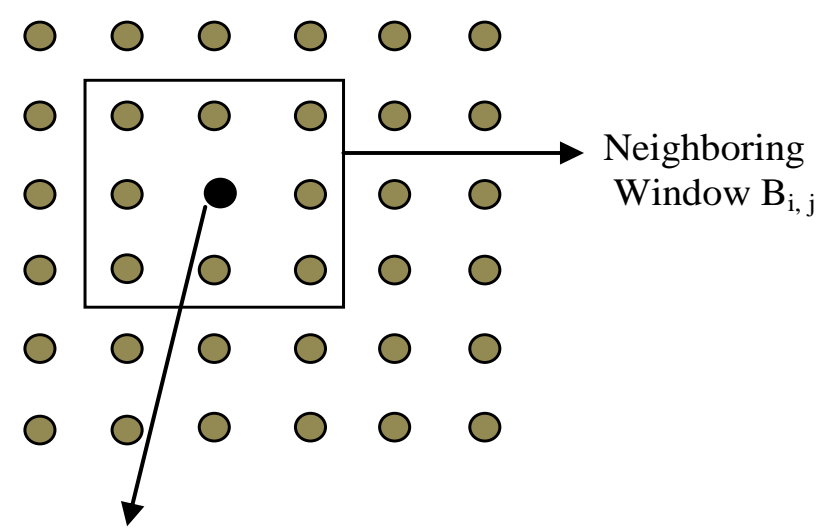

Coefficient for the thresholding $\left(\mathrm{w}_{\mathrm{i}, \mathrm{j}}\right)$

Fig- 10: Representation of surrounding pixel with a window using neigh shrink

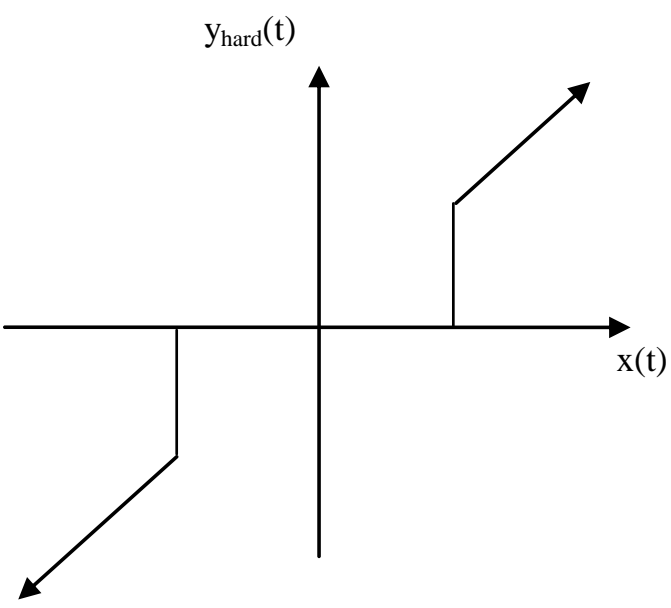

Fig.- 11: Hard thresholding function

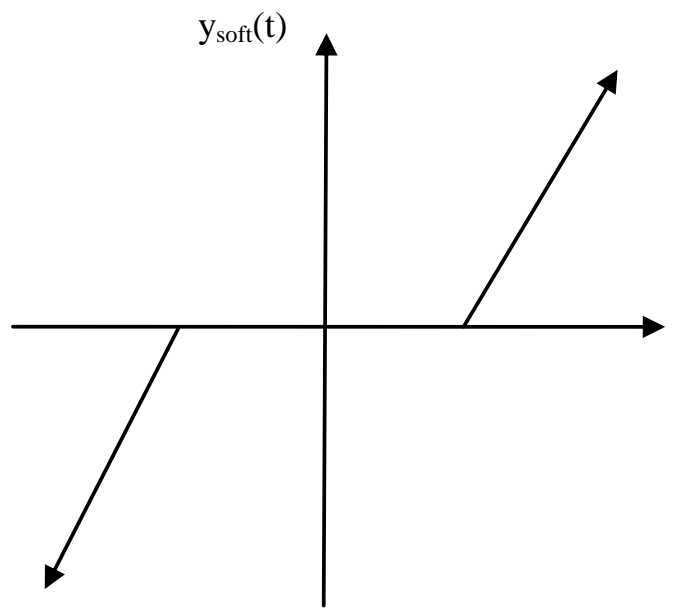

Fig. -12: Soft thresholding function

The hard thresholding may be defined as the process which nullifies the elements or set to zero if their value is below threshold as shown in Fig. 12. Soft thresholding is an extension to the hard thresholding, which tries to bring the elements below the threshold to their parsing limit so they can be preserved as shown in Fig. 11. Each of these techniques has its merits and demerits. Hard thresholding will preserve the curves of the image, but will not be able to get most of the noise out of the image while the soft thresholding will be more effective against the noise, but will result in smoothening of the edges.

The hard thresholding is given by,

$h(i, j)=h(i, j) \quad$ if $h(i, j)>\delta$

The soft thresholding is given by,

$h(i, j)=\operatorname{sgn}(h(i, j) * \max |h(i, j)|)-\delta$

Where $\mathrm{i}$ and $\mathrm{j}$ denotes the location of co-ordinates where pixel is present, $h$ is the value of coefficient and $\delta$ is the threshold for soft and hard thresholding. The neigh shrink stands for neighborhood shrink method. In neigh shrink, we first create a window around the pixel under consideration. 
Then, by considering the window we calculate a threshold which will be applied to every pixel in the window.

Let $g=\{$ gij $\}$ be the noise contaminated signal represented in the matrix form. For the wavelet coefficients, $w=\{w g\}$ indicate the matrix of wavelet coefficients. For every value of wij which is pixel under consideration, let $\mathrm{Bij}$ is a neighboring window around wij, where wij indicates the coefficient under consideration with co-ordinates. $i$ and $j$ denotes co-ordinate location of pixel. The surrounding mesh value can be shown as $\mathrm{L} \times \mathrm{L}$, where $\mathrm{L}$ is a positive odd number. A $3 \times 3$ window mesh centering around the pixel is shown in Fig. 10.

Let,

$\mathrm{S}_{\mathrm{i}, \mathrm{j}}=\sum_{(k, l) \in B i j} W_{k l}^{2}$

Where, $\mathrm{k}$ and $\mathrm{l}$ are the co-ordinates in neighboring window.

The summation in Equation 5 will not be applicable if the pixel indices are out of the bound of component limit. The newly calculated shrinked wavelet coefficient is given by,

$w_{i, j}^{\prime}=w_{i, j} \beta_{i, j}$

The shrinkage factor $\beta_{i, j}$ is represented as,

$\beta_{i, j}=\left(1-\mathrm{T}_{\text {uni }} / \mathrm{s}^{2}{ }_{\mathrm{i}, \mathrm{j}}\right)$

The $\mathrm{T}_{\text {uni }}$ is known as the universal threshold and is given by

$\mathrm{T}_{\text {uni }}=\sqrt{2 \sigma^{2} \ln (n)}$

Where $\mathrm{n}$ is the length of signal and $\sigma$ is noise variance of signal. The value of $\sigma$ and $\mathrm{T}_{\text {uni }}$ should be optimally selected so that it should minimize the MSE and increase the PSNR. Fortunately by using steins criteria (1982) the MSE can be estimated in unbiased nature. The improvement in Neigh Shrink is obtained by calculating individual threshold for every pixel surrounding window for different pixels by using the Stein's Unbiased Risk Estimator(SURE).

\section{EXPERIMENTAL RESULTS}

The experimental trials are applied on test images available like Lena, Barbara and Mandrill of resolution $512 \times 512$.The images are taken in gray scale. The type of noise which is added to original test image is of Gaussian nature of different noise levels variance equal to $10,20,25,30$, 35 .Then, the image is decomposed to $2 \mathrm{D}$ by taking wavelet transform. These wavelet coefficients are modified according to neigh shrink with SURE. The final step is to apply inverse wavelet transform so to reconstruct the denoised image from newly obtained modified coefficient. The window size for neigh shrink is taken in this experiment is $3 \times 3$. In each sub-band, individual pixels of the image are denoised according to the proposed method. For measuring the performance of the method, Peak Signal to Noise Ratio (PSNR) is used, which is calculated using the formula:

$\operatorname{PSNR}(\mathrm{db})=10 \log 10(255)^{2} / \mathrm{MSE}$

The threshold of the proposed method in every sub-band was calculated with Eq. 8. We assumed that the noise variance is known. Generally, noise variance is unknown. We have measured the shrinkage factor using Eq. 7. The PSNR measured by Eq. 9 is in decibels (db).

MSE is the mean square error and shows the average square error between clean image and one with error, lower the MSE higher the denoising performance.

It is given by:

$\operatorname{MSE}=\sum_{i=0}^{R} \sum_{j=0}^{C} \frac{(I s(i, j)-I d(i, j))^{2}}{R \cdot C}$

Where, $\mathrm{R}$ and $\mathrm{C}$ are the dimensions of the image, $I s$ is the original basic test image and $I d$ is the denoised reconstructed image. $i$ and $j$ are co-ordinates of the image. The experimental results are shown in Fig 13, 14, 15, 16 and in table 1 .

\subsection{Result Images}

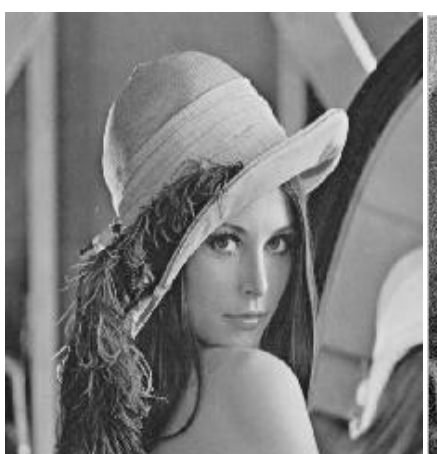

(a)

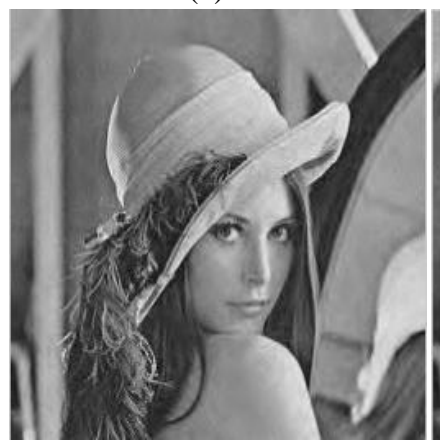

(c)

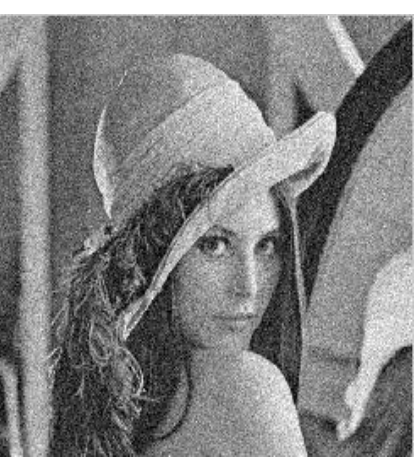

(b)

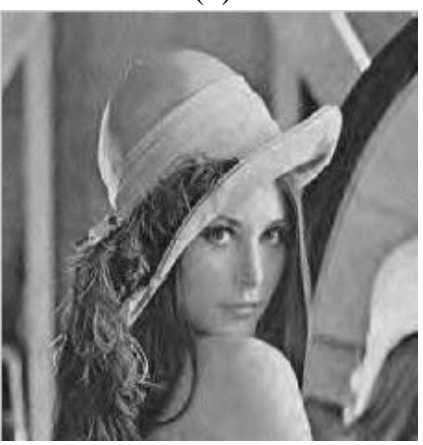

(d)
Fig.- 13: (a) Clean Lena Image (b) Noisy image at $\sigma=20$

(c) Denoised image at $\sigma=20$ (d) Denoised image at $\sigma=30$ 


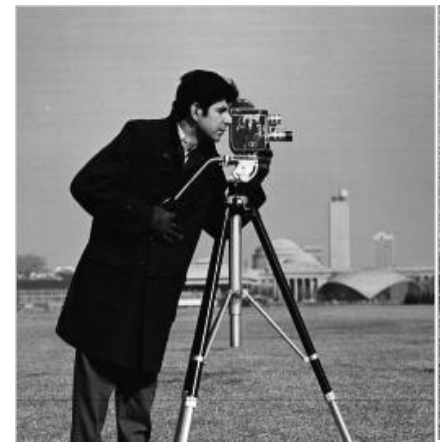

(a)

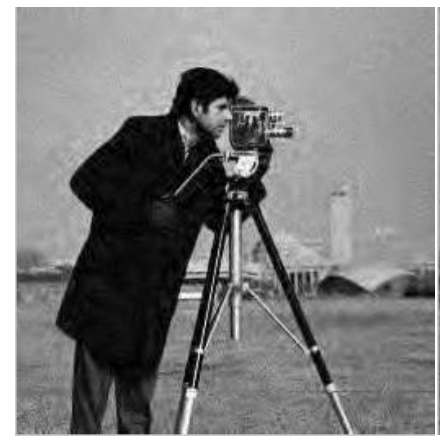

(c)

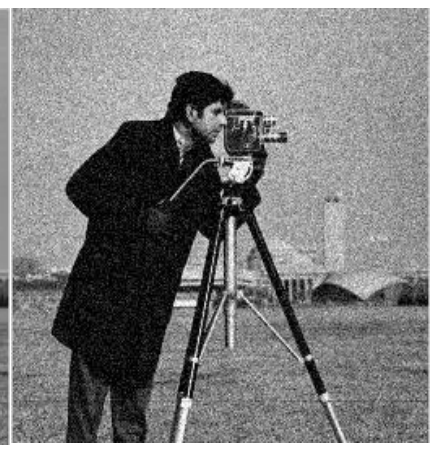

(b)

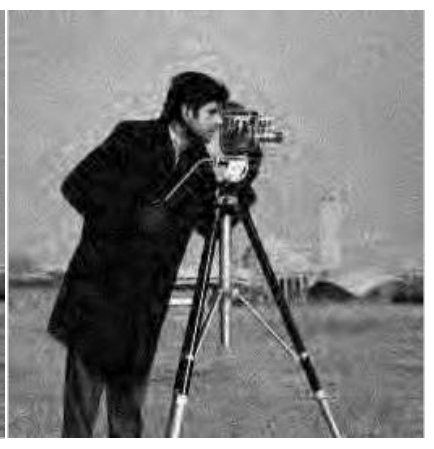

(d)
Fig.- 14: (a) Clean Image (b) Noisy image at $\sigma=20$ (c) Denoised image at $\sigma=20$ (d) Denoised image at $\sigma=30$

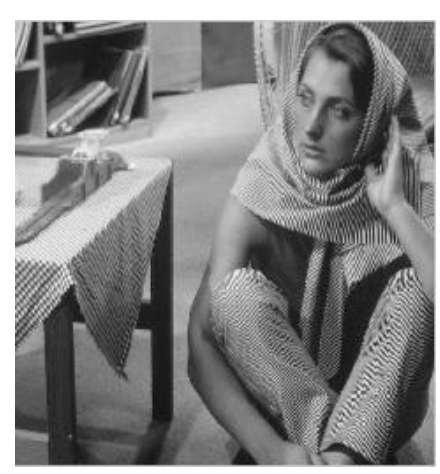

(a)

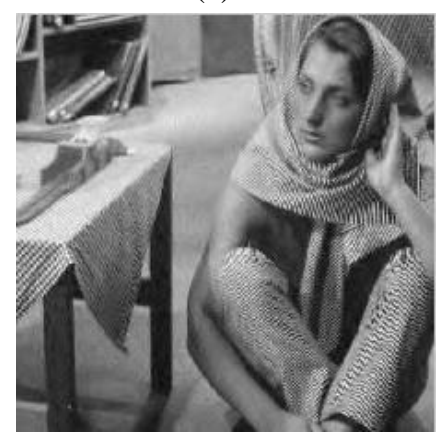

(c)

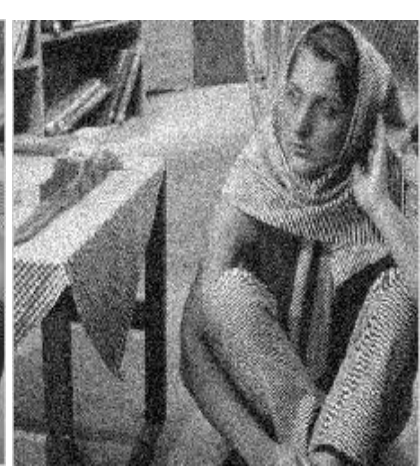

(b)

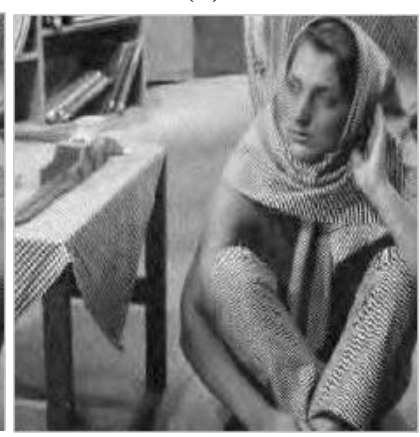

(d)
Fig.- 15: (a) Clean Image (b) Noisy image at $\sigma=20$ (c) Denoised image at $\sigma=20$ (d) Denoised image at $\sigma=30$

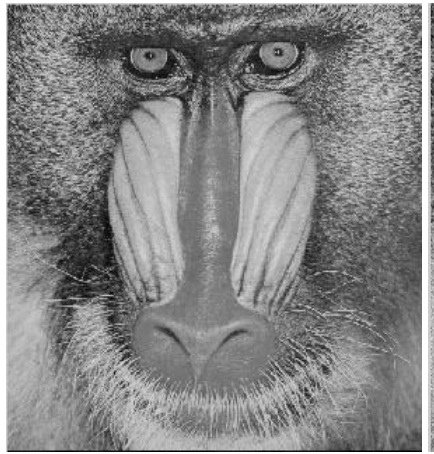

(a)

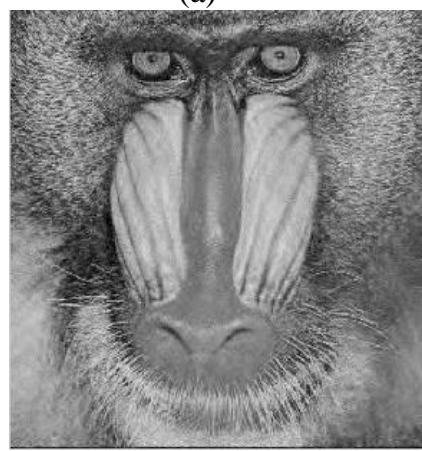

(c)

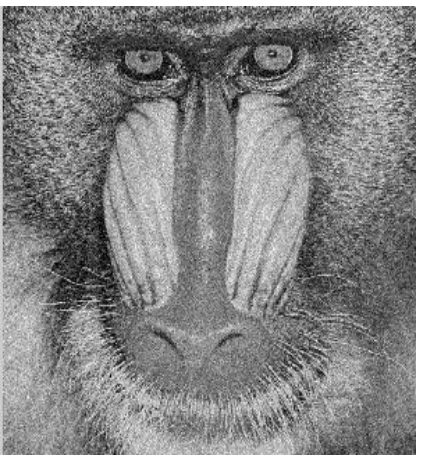

(b)

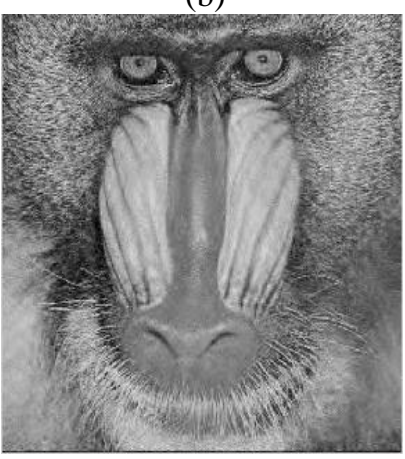

(d)
Fig.- 16: (a) Clean Image (b) Noisy image at $\sigma=20$

(c) Denoised image at $\sigma=20$ (d) Denoised image at $\sigma=30$

Table -1: Neigh shrink based denoising performance

\begin{tabular}{|c|c|c|c|c|}
\hline $\begin{array}{l}\text { Sr. } \\
\text { no. }\end{array}$ & Image & $\begin{array}{c}\text { Variance } \\
(\sigma)\end{array}$ & $\operatorname{PSNR}(\mathrm{db})$ & $\operatorname{MSE}(\mathrm{db})$ \\
\hline \multirow{5}{*}{1} & \multirow{5}{*}{ Lena } & 10 & 34.7204 & 21.9303 \\
\hline & & 20 & 31.5302 & 45.7149 \\
\hline & & 25 & 30.5339 & 57.5025 \\
\hline & & 30 & 29.7023 & 69.6393 \\
\hline & & 35 & 29.0145 & 81.5892 \\
\hline \multirow{5}{*}{2} & \multirow{5}{*}{ Cameraman } & 10 & 32.6388 & 35.4163 \\
\hline & & 20 & 28.4998 & 91.8542 \\
\hline & & 25 & 27.3397 & 119.9742 \\
\hline & & 30 & 26.4033 & 148.8489 \\
\hline & & 35 & 25.6625 & 176.5325 \\
\hline \multirow{5}{*}{3} & \multirow{5}{*}{ Barbara } & 10 & 33.0243 & 32.4076 \\
\hline & & 20 & 29.0935 & 80.1185 \\
\hline & & 25 & 27.9319 & 104.6857 \\
\hline & & 30 & 27.0058 & 129.6403 \\
\hline & & 35 & 26.2530 & 154.0915 \\
\hline \multirow{5}{*}{4} & \multirow{5}{*}{ Mandrill } & 10 & 30.3021 & 60.6554 \\
\hline & & 20 & 26.1965 & 156.1099 \\
\hline & & 25 & 25.0418 & 203.6582 \\
\hline & & 30 & 24.1647 & 249.2378 \\
\hline & & 35 & 23.4666 & 292.6986 \\
\hline
\end{tabular}




\section{CONCLUSION}

In this research paper the image denoising using wavelet transform is successfully implemented. Compared with Neigh Shrink, the proposed method can individually calculate separate threshold by using SURE algorithm and surrounding mesh value for each and every wavelet subcomponent instead of applying same universal threshold across all pixels for the same surrounding window size in all sub-components. The proposed technique is a general purpose algorithm and is effective in eliminating any kind of noise like gaussian, speckled or salt pepper corrupting the image. The algorithm is also applicable for different types of images.

\section{REFERENCES}

[1] Mallat S, Hwang W L. Singularity detection and processing with Wavelets [J]. IEEE Transactions on Information Theory, 1992, 38 (2) : 617 643.

[2] Xu Y, Weaver B, Healy D M, et al. Wavelet transform domain filters: A spatially selective noise filtration technique [J]. IEEE Transactions on Image Processing, 1994, 3(6) : 217 237.

[3]Donoho D L, John stone IM. Ideal spatial adaptation via wavelet shrinkage [J]. Biometrika, 1994, 81 (3) : 425 455.

[4] DONOHO D L. De-Noising by Soft-Threshold .IEEE Transactions on Information Theory, 1995, 41(3):613-627.

[5] D.L.Donoho and I.M.John stone. Adapting to Unknown Smoothness via Wavelet Shrinkage [J]. Journal of American StatAssoc, vol.12, pp.1200- 1224, 1995

[6] S.Arivazhagan, S.Deivalakshmi, K.Kannan,Performance Analysis of Image Denoising System for different levels of Wavelet decomposition," International Journal of Imaging Science and Engineering (IJISE), Vol.1, No.3, July 2007.

[7] Rakesh Kumar and B.S.Saini,"Improved Image Denoising Techniques Using Neighbouring Wavelet Coefficients of Optimal Wavelet with Adaptive Thresholding," International Journal of Computer Theory and Engineering, Vol.4, No.3, June 2012.

\section{BIOGRAPHIES}

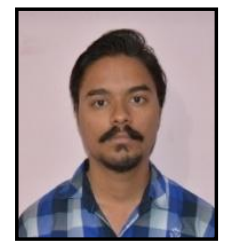

Mr. Sameer Khedkar is pursuing M.Tech. in Communication Engineering 2nd year from G. H. Raisoni College of Engineering Nagpur.

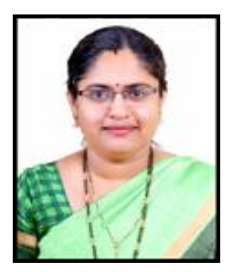

Dr. Kalyani Akant did her B.E and M.Tech in Electronics Engineering in 1994 and 2005 respectively. She received her $\mathrm{Ph} . \mathrm{D}$. degree in Electronics Engineering in 2014 from RTM Nagpur University, Nagpur, India. Her research area is Digital Signal Processing, Image Processing and Music Signal Processing.

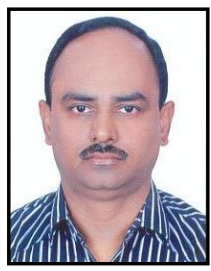

Dr. Milind Khanapurkar is a professor and head of the department of Electronics and Telecommunication Engineering. He has 25 years of teaching and research experience with around 30 research contributions with book chapters. He has one patent on his name with and is recipient of several grants from various funding agencies. 